\title{
Serialization of Simultaneity in Mandarin
}

\author{
LIANCHENG CHIEF \\ University at Buffalo, The State University of New York
}

\section{Introduction}

To express that two events occur concurrently, the "Agent $\mathrm{VP}_{1}$-zhe $\mathrm{VP}_{2}$ " construction is often used in Mandarin, as shown in (1).

(1) ta zuo zhe du shu

he sit DUR read book

'He read a book while sitting.'

$\begin{array}{rllll}* \text { ta } & \text { du } & \text { zhe } & \text { shu } & \text { zuo } \\ \text { he } & \text { read } & \text { DUR } & \text { book } & \text { sit }\end{array}$

In (1) and (2) the two events, sitting and reading, overlap temporally, but only one sequential arrangement is allowed. This paper shows that this asymmetry can be determined solely by the semantic properties of the verbs involved in terms of Figure and Ground event assignment (Talmy 2000).

This paper focuses on the following questions. First, given two verbs that can be used to describe two concurrent events, can we predict which one is realized as the subordinate $\mathrm{VP}_{1}$ and which one is realized as the main $\mathrm{VP}_{2}$ in the "Agent $\mathrm{VP}_{1}$-zhe $\mathrm{VP}_{2}$ " construction? Second, if we can make such predictions, what is the motivation behind this pattern? This paper claims that the correlation between Figure and Ground events and main and subordinate VPs is the answer.

This paper is organized as follows. Section 1 gives some background information about the "Agent $\mathrm{VP}_{1}$-zhe $\mathrm{VP}_{2}$ "construction. Section 2 demonstrates that some verbs are likely to be expressed as $\mathrm{VP}_{1}$ or $\mathrm{VP}_{2}$ on the basis of corpus data. ${ }^{1}$ Section 3 shows that the distinction between Figure and Ground events correlates well with the distinction between main and subordinate verb phrases in this construction and that this correlation accounts for the data discussed in section 2. The final section concludes the paper.

\footnotetext{
${ }^{1}$ Most of the data used in this study are taken from the Sinica Corpus. Information on the Sinica Corpus 3.0 can be found at http://www.sinica.edu.tw/ftms-bin/kiwi.sh.
} 


\section{Liancheng Chief}

\section{The "Agent $\mathrm{VP}_{1}-$ zhe $\mathrm{VP}_{2}$ " Construction}

The "Agent $\mathrm{VP}_{1}$-zhe $\mathrm{VP}_{2}$ " construction in Mandarin can be used to describe two concurrent events. In this construction, the durative marker -zhe is attached to the first verb. Traditionally, the first VP is analyzed as the subordinate VP while the second VP is the main VP (Chu 1987). An intriguing fact about this construction is that the sequential arrangement of the two VPs is not random but governed by certain principles. Take sentences (1) and (2), for example: the two events, sitting and reading, occur simultaneously, but only one sequential arrangement of the two events is allowed, namely, the sitting event is expressed as subordinated to the main event reading. If the encoding of events is reversed, then the sentence is ungrammatical as (2) shows.

One might suggest that there is a lexical or syntactic constraint that certain verbs can only occur in the first verb or second verb position. The following three sentences prove it is not the case.

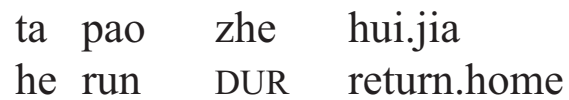

'He went home running.'

(4) ta chuan zhe xie pao

he wear DUR shoes run

'He ran with shoes on.'

$$
\begin{aligned}
& \text { ta zhan zhe chuan yifu } \\
& \text { he stand DUR wear clothes } \\
& \text { 'He wore clothes while standing.' }
\end{aligned}
$$

The verb pao 'run' can appear either in $\mathrm{VP}_{1}$ position as in (3) or $\mathrm{VP}_{2}$ position as in (4). Similarly, the verb chuan 'wear' can occur either in $\mathrm{VP}_{1}$ as in (4) or $\mathrm{VP}_{2}$ as in (5). Obviously, there is no particular constraint against certain verbs occurring in $\mathrm{VP}_{1}$ or $\mathrm{VP}_{2}$. However, if the order of the two verbs in each sentence is reversed, all sentences in (3) through (5) are unacceptable. This suggests that the order of VPs is relative; it depends on the pair of verbs involved. I will argue that the order follows from how Figure and Ground events (Talmy 2000) are expressed in this construction. This approach provides a semantic motivation that previous analyses neglect to offer. For example, Chen (1986) asserts that the interrelationship between the two VPs in this construction has to be conventional, logical, and physical. Furthermore, $\mathrm{VP}_{2}$ has to have predominant weight. These semantic notions are not fully explained and remain vague. I will show that the concept of Figure and Ground events can provide a simple and unified account of the order of $\mathrm{VP}_{1}$ and $\mathrm{VP}_{2}$.

\section{Verbs That Tend to Occur in $V P_{1}$ and $V P_{2}$}

The first question this paper focuses on is whether, given two verbs that can be 


\section{Serialization of Simultaneity in Mandarin}

used to describe two concurrent events, we can predict which one is realized as the subordinate $\mathrm{VP}_{1}$ and which one is realized as the main $\mathrm{VP}_{2}$. To answer this question, I looked at corpus data.

Based on my preliminary study of the Sinica Corpus, ${ }^{2}$ some verb classes tend to occur in the $\mathrm{VP}_{1}$ position or $\mathrm{VP}_{2}$ position. The figure in (6) is the result of my study of the Sinica Corpus. In (6), verb classes are shown in pairs. In each pair, the one to the left appears as $\mathrm{VP}_{1}$ when it co-occurs with verb classes on the right side. For example, when a posture verb occurs with a motion verb, the posture verb is always realized as $\mathrm{VP}_{1}$ and the motion verb as $\mathrm{VP}_{2}$. Similarly, when facial expression verbs co-occur with either communication verbs or translocation verbs, it is the facial expression verb that is realized as the subordinate $\mathrm{VP}_{1}$.

The Relative Occurrence of $\mathrm{VP}_{1}$ and $\mathrm{VP}_{2}$ in Pairs of Verbs

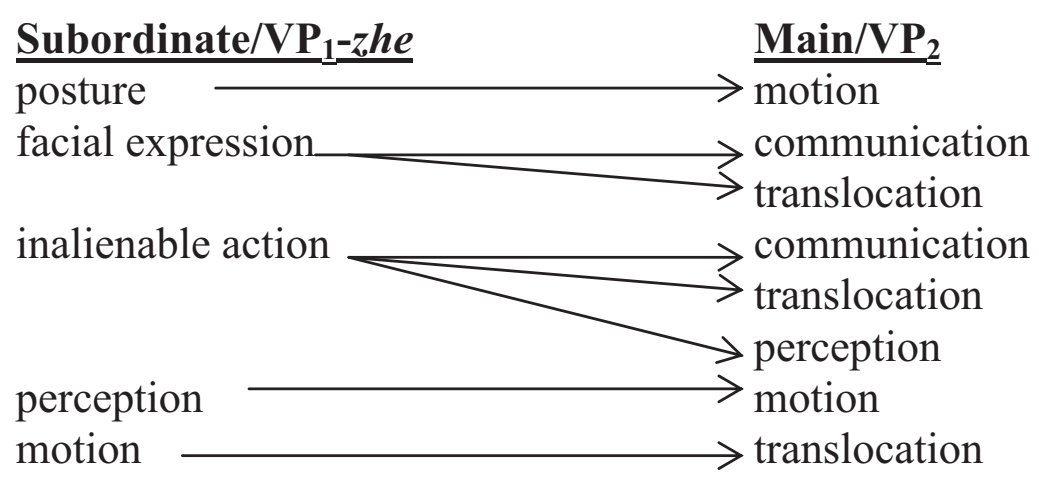

Members of each verb class in (6) are listed in (7).

(7) a. Posture verbs

i: Manner of posture: quanqu 'curl'

ii: Posture: zuo 'sit', zhan 'stand', tang 'lie', dun 'squat'

b. Verbs involving the body

i: Facial expression: $k u$ 'cry', xiao 'smile'

ii: Inalienable action: rou yanjing 'rub eyes', yao tou 'shake one's head', hui shou 'wave one's hand', chui koushao 'whistle'

c. Composite

There is a static spatial relation between the subject and the object of the verb: kang 'carry something on one's shoulder', bao 'hold', chuan 'wear'

d. Perception

i. Inactive: ting 'listen'

ii. Active: $d u$ 'read', kan 'watch'

\footnotetext{
2 The data are extracted from the Sinica Corpus website, which randomly gives 2,000 results at most. My observation is based on sentences which contain the "Agent $\mathrm{VP}_{1}-z h e \mathrm{VP}_{2}$ " constructions among these 2,000 sentences.
} 


\section{Liancheng Chief}

e. Motion

kai che 'drive a car', pao 'run', zou 'walk'

f. Communication

shuo 'speak, talk', wen 'ask'

g. Translocation

likai 'leave', jin 'enter', dao 'arrive'

In the rest of this section, I provide some examples that illustrate (6). In (8) through (10), all posture verbs are realized as $\mathrm{VP}_{1}$. If the order of the verb phrases is reversed, the sentences become ungrammatical.

(8) ta tang zhe ting yinyue

he lie DUR listen music

'He listened to the music while lying.'

(9) ta zuo zhe zhaogu yang.qun

she sit DUR look.after herd.of.sheep

'She looked after a herd of sheep while sitting.'

(10) ta zhan zhe chang ge

he stand DUR sing song

'He sang while standing.'

(6) shows that facial expression verbs tend to be $\mathrm{VP}_{1}$ when they co-occur with verbs of communication or translocation. This is illustrated by sentences (11) and (12).

(11) ta $\mathrm{ku}$ zhe pao.hui jia

he cry DUR run.return home

'He ran home crying.'

(12) ta weixiao zhe wen xuesheng wenti

he smile DUR ask student question

'He asked students questions smiling.'

The following set of sentences contains verbs describing how an agent acts on himself, e.g., rubbing his eyes or shaking his head.

(13) ta yao zhe tou zou.kai

he shake DUR head walk.away

'He walked away shaking his head.' 


\section{Serialization of Simultaneity in Mandarin}

(14) ta rouzhe yanjing shuohua
he rub DUR eyes talk
'He talked rubbing his eyes.'

In (13) and (14), verbs involving bodily actions are realized as $\mathrm{VP}_{1}$ when they co-occur with communication, translocation, or perception verbs. If the order of each verb phrase is reversed, the resulting sentences are ungrammatical.

Example (15) illustrates a situation in which a perception verb and an activity verb occur at the same time. In this situation, the perception verb occurs in $\mathrm{VP}_{1}$.

$$
\begin{aligned}
& \text { ta ting zhe yinyue xie gongke } \\
& \text { he listen DUR music write homework } \\
& \text { 'He wrote homework listening to music.' }
\end{aligned}
$$

The general tendency is clear: some verbs have to occur within $\mathrm{VP}_{1}$ relative to other verbs. As the next section shows, the data presented in this section can be accounted for by a general principle, i.e., the assignment of Figure and Ground events to main and subordinate clauses.

\section{Figure and Ground Event Correlation}

Talmy (2000) indicates that Figure and Ground are central to the conceptual organization of languages. The Ground functions as a reference point for locating the Figure in space. Because of a spatiotemporal homology in language, these two concepts can also be applied to explain the patterns of relative location of events in time. Talmy demonstrates that the Figure event is realized in the main clause and the Ground event is realized in the subordinate clause. He uses while clauses to illustrate this claim. Hayase (1997) also mentions that in Japanese the Figure and Ground events are aligned to main clauses and subordinate clauses, respectively. Both the English and the Japanese examples in their studies are complex sentences with two clauses, in the sense that the subordinate clause is marked explicitly by while or nagara, respectively. In the Mandarin construction at issue in this paper, the relation between two VPs is tighter, in the sense that they are not composed of two clauses and there is no overt subordinate clause marker. In structural terms, the first VP is subordinated to the second VP, which is treated as the main VP. If we extend Talmy's proposal that in a complex sentence the Figure event is expressed by the main clause and the Ground event is expressed by the subordinate clause, then we can set up the following correlation for the Mandarin "Agent $\mathrm{VP}_{1}-z h e \mathrm{VP}_{2}$ " construction.

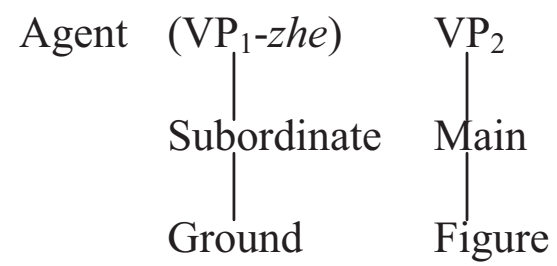




\section{Liancheng Chief}

(16) indicates that $\mathrm{VP}_{1}$ is the subordinate $\mathrm{VP}$ and $\mathrm{VP}_{2}$ the main VP. Moreover, $\mathrm{VP}_{1}$ is the Ground event and $\mathrm{VP}_{2}$ the Figure event. It is generally agreed that the first VP is subordinate to the second VP in the "Agent $\mathrm{VP}_{1}-z h e \mathrm{VP}_{2}$ " construction (Li and Thompson 1981). Therefore, what remains to be examined is which VPs encode the Figure and Ground events.

There are some basic characteristics associated with the concept of Figure and Ground events. Generally, the Figure denotes an event which describes a more salient occurrence with respect to the Ground, which denotes a reference event that has a stationary setting. The following example illustrates this tendency.

$\begin{array}{llll}\text { ta cheng zhe } & \text { yusan } & \text { zou } \\ \text { he hold } & \text { DUR } & \text { umbrella } & \text { walk }\end{array}$

'He walked holding an umbrella.'

$$
\begin{array}{rlll}
* \text { ta } & \text { zou } & \text { zhe } & \text { cheng yusan } \\
\text { he walk } & \text { DUR } & \text { hold umbrella }
\end{array}
$$

The walking event expresses the notion of translocation. On the other hand, the event of holding an umbrella depicts a static relationship between the person who holds the umbrella and the umbrella. Since translocation denotes a situation which is more salient than static, the walking event should be categorized as the Figure event and the event of holding an umbrella should be categorized as the Ground event. This is why sentence (17) is good and sentence (18) is odd. In (18), the walking event is assigned Ground event status as it is encoded as a subordinate VP, despite its higher dynamicity; hence the ungrammaticality of the sentence. This set of examples shows that generally Figure status should be assigned to the main $\mathrm{VP}_{2}$ and Ground status to the subordinate $\mathrm{VP}_{1}$. More generally, we can summarize that if certain verbs tend to occur within $\mathrm{VP}_{1}$ relative to their collocating verbs, these verbs tend to be assigned Ground status and vice versa if they tend to occur within $\mathrm{VP}_{2}$.

In the following, I discuss several constraints that distinguish Figure events from Ground events. Section 3.1 examines the contrast between point events and extent events. Section 3.2 shows events that depict situations in which the location of entities changes or is more permanent. Section 3.3 examines the contrast between more salient and more backgrounded events. Based on these associated characteristics of Figure and Ground, I show that, given two events that are used to describe temporally overlapping events, one is more likely to be the Figure than the other, and that this distinction, in turn, can account for the examples mentioned in section 2 .

\subsection{Extent vs. Point Events}

One of the associated characteristics of Figure and Ground is that one is geometrically simpler (often point-like), and the other is geometrically more complex. In terms of temporality, the Figure event is more a point-like event and 


\section{Serialization of Simultaneity in Mandarin}

the Ground event is an extent event. In other words, Figure events tend to be telic, whereas Ground events tend to be atelic. In the following examples, all the main VPs which express Figure events are telic.
ta pao zhe hua.jia
he run DUR return.home

'He went home running.'

(20) ta yao zhe tou zou.kai

he shake DUR head walk.away

'He walked away shaking his head.'

(21) ta kang zhe futou zou.jin linjian

he carry DUR axe walk.enter woods

'He walked into the woods carrying an axe.'
tamen hua zhe chuan dao hehuacong li
they row DUR boat arrive lotus.cluster in
'They entered into the lotus clusters rowing the boat.'

For each sentence, the order of the two VPs cannot be reversed. Otherwise, the sentence is ungrammatical. For each pair of verbs, one is an atelic verb and the other is a telic verb. For example, pao 'run' is atelic whereas hui.jia 'return home' is telic. The sequential arrangement in these examples supports the claim that the Figure event is realized as the main VP, and the Ground event is realized as the subordinate VP. When a telic verb and an atelic verb are used to describe concurrent events, the telic verb is assigned the Figure event status and the atelic verb the Ground one. In the "Agent $\mathrm{VP}_{1}-z$ he $\mathrm{VP}_{2}$ " construction, the telic verb is expressed as the main $\mathrm{VP}_{2}$ and the atelic verb as the subordinate $\mathrm{VP}_{1}$. This correlation supports the mapping between Figure/Ground Event and Main/Subordinate VP in (7).

\subsection{More Permanently Located vs. More Movable}

There are cases where one event describes a more permanently located relation between two entities and the other event describes a more movable relation between two entities. In this case, the former one is categorized as the Ground and the latter one as the Figure. One manifestation of this relation is when the subject of the whole sentence and the object of the VP form a Composite Figure. The following example taken from Talmy (2000) illustrates this situation.

(23) The lion chased the gazelle through the forest.

In this sentence, there are multiple levels of Figure and Ground relationships. Specifically, the gazelle in (23) has two statuses. With respect to the lion, the 


\section{Liancheng Chief}

Figure, it is the Ground. However, if they move at the same speed, then their Figure and Ground relation is static. In this sense, both of them form a Composite Figure with respect to the forest, which is the Ground in this relation.

It seems that if the object of a verb can form a Composite Figure with the subject, then this verb has to be expressed as the first verb in the "Agent $\mathrm{VP}_{1}$-zhe $\mathrm{VP}_{2}$ " construction, when it co-occurs with translocational verbs. The following examples all illustrate this tendency. In these sentences, the subject and the object of the first verb form a Composite Figure because their Figure and Ground relation is static. In other words, $\mathrm{VP}_{1}$ in (24) through (27) describes a static event; hence the first VP is the Ground event.

ta bao zhe baobao pao
he hold DUR baby run
'He ran holding a baby.'

ta kang zhe futou zou.jin linjian

he carry DUR axe walk.enter woods

'He walked into the woods carrying an axe.'

tamen hua zhe chuan dao hehua.cong li
they row DUR boat arrive lotus.cluster into
'They entered into the lotus clusters rowing the boat.'
ta chuan zhe xie pao
he wear DUR shoes run
'He ran with shoes on.'

In (24), the relation between the baby-holder and the baby is static. This is also true for the axe-carrier and the axe in (25), the boat-rower and the boat in (26), and the runner and her shoes in (27). Their relation is static because the two entities in question move together. Other verbs that co-occur with them describe events that involve more motion such as translocation, i.e., the Composite Figure moves from one place to the other in space with respect to the Ground. For instance, in (25), the axe-carrier and the axe form a Composite Figure with respect to the woods which now functions as the Ground. Similarly, in (26), the boat-rower and the boat form a Composite Figure and the lotus cluster is the Ground. To sum up, given two verbs in this construction, if the object of these verbs can form a Composite Figure with the subject, then this verb has to be realized as the subordinate $\mathrm{VP}_{1}$. This is semantically motivated because this verb describes a static relation and this relation is more likely to receive Ground event status. Here again, the assignment of Figure and Ground event status is determined by the semantic properties of each verb. 


\section{Serialization of Simultaneity in Mandarin}

\subsection{More Backgrounded vs. More Salient Events}

It has already been noted in the literature that in the "Agent $\mathrm{VP}_{1}$-zhe $\mathrm{VP}_{2}$ " construction, the first VP functions as the background for the meaning of the second VP. For example, Tai (1993) says that the first VP provides backgrounded information and the second VP provides foregrounded information. In addition, Chu (1987) points out that $-z$ he is "a durative aspect marker in semantics, a subordinating suffix in syntax and a backgrounding device in pragmatics." However, why one meaning must be backgrounded relative to the other has not been discussed. The distinction between Figure and Ground events motivates this constraint.

$$
\begin{aligned}
& \text { ta xiao zhe shuo.hua } \\
& \text { he smile DUR talk } \\
& \text { 'He talked with a smile.' }
\end{aligned}
$$

For example, in sentence (28), the smiling event is treated as the background event. However, given that $-z h e$ can attach to both verbs, xiao and shuo, why is the following sentence ungrammatical?

$$
\begin{array}{cllll}
\text { *ta shuo } & \text { zhe } & \text { hua } & \text { xiao } \\
\text { he talk } & \text { DUR } & \text { word } & \text { smile }
\end{array}
$$

(29) shows that the talking event cannot be backgrounded when it occurs concurrently with the smiling event. Note that shuo can occur in the first VP or be backgrounded too when it co-occurs with other verbs, as shown in (30).

$$
\begin{aligned}
& \text { ta shu zhe huazoulu } \\
& \text { he talk DUR talkwalk } \\
& \text { 'He walked talking.' }
\end{aligned}
$$

These examples demonstrate that we need to predict what factors determine which event is backgrounded. In that respect, it is important to note that what is the Figure event and what is the Ground event is relative. Specifically, the talking event is interpreted as the Figure event relative to the smiling event, and is interpreted as the Ground event relative to the walking event. By comparing smiling and talking, we can see that talking has more Figure-associated features. For example, talking is more salient and of greater concern than smiling. In other words, when a person is smiling and talking at the same time, people pay more attention to what she is talking about. Therefore, in (28) the talking event has to be categorized as the Figure. In (30) the walking involves more motion than the talking event and therefore receives the Figure event status. Thus, while I agree with previous analyses that the first VP is backgrounded relative to the second VP, I claim that the motivation behind this difference lies in the relative categorization of the two events as Figure and Ground. In brief, Ground events are more likely to 


\section{Liancheng Chief}

be backgrounded.

In this section, I discussed how the assignment of Figure and Ground events is determined, and its application to the data presented in section 2. The assignment can be determined by the telicity of verbs or by the lexical semantics of verbs in the sense that whether two arguments of a verb can form a Composite Figure or not is determined lexically. To sum up, for a pair of verbs, if one of them is associated with more Figure or Ground event characteristics, then they are categorized as the Figure or Ground event. Furthermore, based on Talmy's claim that in complex sentences Figure events are expressed by main clauses and Ground events by subordinate clauses, we can predict the arrangement of $\mathrm{VP}_{1}$ and $\mathrm{VP}_{2}$ in the "Agent $\mathrm{VP}_{1}$-zhe $\mathrm{VP}_{2}$ " construction.

\section{Conclusion}

This paper discusses the Mandarin "Agent $\mathrm{VP}_{1}-z h e \mathrm{VP}_{2}$ " construction in terms of Talmy's distinction between Figure and Ground events. It shows that given two verbs which describe temporally overlapping events, it is possible to predict which verb is realized as part of $\mathrm{VP}_{1}$ or part of $\mathrm{VP}_{2}$. Based on corpus data I have shown that some verbs tend to occur in either $\mathrm{VP}_{1}$ or $\mathrm{VP}_{2}$ position. In addition, in this construction, $\mathrm{VP}_{1}$ is categorized as the Ground event and $\mathrm{VP}_{2}$ as the Figure event. By comparing two co-occurring verbs, relative Figure and Ground assignment is determined on the basis of the semantic properties of each verb in the pair. Once the Figure and Ground event assignment is determined, the asymmetry between the two VPs in this construction can be predicted and accounted for.

\section{Acknowledgement}

I would like to thank Jean-Pierre Koenig, Leonard Talmy, and Hui-Chen Sabrina Hsiao for their comments. All remaining errors are mine.

\section{References}

Chen, Chung-yu. 1986. Constraints on the 'V1-zhe...V2' structure. Journal of Chinese Language Teachers Association 21(1):1-20.

Chu, Chauncey. 1987. The semantics, syntax, and pragmatics of the verbal suffix -zhe. Journal of Chinese Language Teachers Association 22(1):1-41.

Hayase, Naoko. 1997. The role of Figure, Ground, and coercion in aspectual interpretation. In M. Verspoor, K. D. Lee, and E. Sweetser, eds., Lexical and Syntactical Constructions and the Construction of Meaning, 33-50. Amsterdam: John Benjamins.

Li, Charles N., and Sandra A. Thompson. 1981. Mandarin Chinese. Berkeley and Los Angeles: University of California Press. 
Tai, James H-Y. 1993. Iconicity: Motivations in Chinese grammar. In M. Eid and G. K. Iverson, eds., Principles and Prediction: The Analysis of Natural Language. Papers in Honor of Gerald Sanders, 153-174. Amsterdam: John Benjamins.

Talmy, Leonard. 2000. Toward a Cognitive Semantics. Cambridge, MA: MIT Press.

Linguistics Department

609 Baldy

University at Buffalo, The State University of New York

Buffalo, NY 14260

lchief@buffalo.edu 